\title{
Reversible Complex Hénon Maps
}

\author{
C. R. Jordan, D. A. Jordan, and J. H. Jordan
}

\section{CONTENTS}

1. Introduction

2. Reversibility

3. Fixed Points

4. Periodic Points of Order 2

5. Dynamical Relations

6. Orbits

References
2000 AMS Subject Classification: Primary 32H50, 37F10;

Secondary 37C25, 37E15, 37F45

Keywords: Hénon map, reversibility, fixed points, periodic points, bounded orbits, ellipticity
We identify and investigate a class of complex Hénon maps $H$ : $\mathbb{C}^{2} \rightarrow \mathbb{C}^{2}$ that are reversible, that is, each $H$ can be factorized as $R U$ where $R^{2}=U^{2}=\operatorname{Id}_{\mathbb{C}^{2}}$. Fixed points and periodic points of order two or three are classified in terms of symmetry, with respect to $R$ or $U$, and as either elliptic or saddle points. We report on experimental investigation, using a Java applet, of the bounded orbits of $H$.

\section{INTRODUCTION}

For $\alpha, \beta \in \mathbb{C}$, the Hénon map $H_{\alpha, \beta}: \mathbb{C}^{2} \rightarrow \mathbb{C}^{2}$ is defined by the rule

$$
H_{\alpha, \beta}((z, w))=\left(\alpha-\beta w-z^{2}, z\right) .
$$

If $\alpha, \beta \in \mathbb{R}$, then $H_{\alpha, \beta}$ restricts to the real Hénon map $H_{\alpha, \beta}: \mathbb{R}^{2} \rightarrow \mathbb{R}^{2}$. Real and complex Hénon maps, and their history, are well-documented, see, for example, [Devaney 89, Hale and Koçak 91] for the real case, and [Friedland et al. 89, Bedford et al. 91, Bedford et al. 93, Hubbard and Oberste-Vorth 94, Oberste-Vorth 97, Smillie and Buzzard 97] for the complex case.

If $R$ is an involution of $\mathbb{R}^{n}$ and $F: \mathbb{R}^{n} \rightarrow \mathbb{R}^{n}$ or if $R$ is an involution of $\mathbb{C}^{n}$ and $F: \mathbb{C}^{n} \rightarrow \mathbb{C}^{n}$ then, following [Devaney 76, Devaney 84], $F$ is $R$-reversible if $F^{-1}=R F R$. This is equivalent to requiring that $R F$ is an involution or that $F=R U$ for some involution $U$.

Let $R$ and $S$ denote the involutions of $\mathbb{C}^{2}$ such that $R((z, w))=(w, z)$ and $S(z, w)=(-w,-z)$, or, where appropriate, their restrictions to $\mathbb{R}^{2}$. If $\alpha \in \mathbb{R}$, the real Hénon maps $H_{\alpha, 1}$ and $H_{\alpha,-1}$ are $R$-reversible and $S$-reversible, respectively, and are discussed in [Devaney 84] and [Devaney 89, Section 2.9, Exercises 21-34]. The only comment on reversible complex Hénon maps that we have found in the literature is a comment in [Friedland et al. 89], where a conjugate form of $H(\alpha, \beta)$ is used, that $H_{\alpha, \beta}$ is $R$-reversible if and only if $\beta=1$ and $S$-reversible if and only if $\beta=-1$. For $\beta \in \mathbb{C}$, with $|\beta|=1$, let $R_{\beta}$ be the involution of $\mathbb{C}^{2}$ such that

$$
R_{\beta}((z, w))=(\beta \bar{w}, \beta \bar{z})
$$



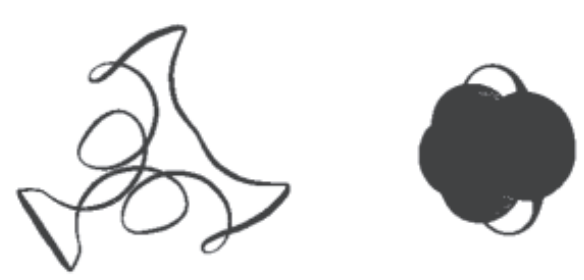

FIGURE 1. Projections of orbits.

Then $R_{1}$ and $R_{-1}$ have the same restrictions to $\mathbb{R}^{2}$ as $R$ and $S$.

In Section 2, we shall see that $H_{\alpha, \beta}$ is $R_{\beta}$-reversible if and only if $\alpha \in \mathbb{R} \beta$. In this case, the involution $R_{\beta} H_{\alpha, \beta}$ is given by $(z, w) \mapsto\left(\beta \bar{z}, \beta \bar{\alpha}-\bar{w}-\beta \bar{z}^{2}\right)$. In [Devaney 76 , Devaney 84] the involutions $R$ are assumed to be diffeomorphisms. Although $R_{\beta}$ is not a $\mathbb{C}$-diffeomorphism of $\mathbb{C}^{2}$, it is a $\mathbb{R}$-diffeomorphism when $\mathbb{C}^{2}$ is identified with $\mathbb{R}^{4}$, so the results of [Devaney 84 ] apply.

The role of the reflection $z \mapsto \beta \bar{z}$ in the involutions $R_{\beta}$ and $R_{\beta} H_{\alpha, \beta}$ gives rise to orbits, with reflective symmetry, that can be quite striking in appearance. Figure 1 shows projections onto the $z$-plane of two examples.

The reversibility not only influences the geometry of orbits but facilitates calculation and analysis. In Section 3, we analyse the fixed points and determine when they are symmetric, for either of the involutions $R_{\beta}$ or $R_{\beta} H_{\alpha, \beta}$, and when they are elliptic. We shall do the same for periodic points of order 2 or 3 in Section 4. Section 5 is concerned with local dynamics and establishes a sufficient condition for an orbit to be unbounded. This has been applied to plot bounded orbits. (A Java applet is available at http://www.shef.ac.uk/ ${ }^{\text {daj} / h e n o n / H . h t m l .) ~ T h e ~ f i n a l ~}$ section reports on experimental observations of such orbits. For example, if $\beta$ is a primitive $m$ th root of unity, then $\operatorname{orb}((0,0))$, if bounded, appears to be dense in the union of $m$ closed curves which are deformations of ellipses, becoming more deformed as $|\alpha|$ increases. We also comment on the influence on orbits of nearby periodic elliptic points and on bifurcation.

Our interest in Hénon maps arose from a problem in [Jordan 93, 3.3], a special case of which would ask whether, for a nonperiodic orbit $\left\{\left(z_{n}, w_{n}\right)\right\}_{n \in \mathbb{Z}}$ of the Hénon map, $z_{n}$ could take the same value infinitely often.

\section{REVERSIBILITY}

Lemma 2.1. Let $\alpha, \beta, \rho \in \mathbb{C}$, with $|\rho|=1$, let $H=H_{\alpha, \beta}$, and let $R_{\rho}$ be the involution of $\mathbb{C}^{2}$ such that $R_{\rho}((z, w))=$
( $\rho \bar{w}, \rho \bar{z})$. Then $H$ is $R_{\rho}$-reversible if and only if $\beta=\rho$ and $\alpha \in \mathbb{R} \beta$.

Proof: It is easily checked that $\left(R_{\rho} H_{\alpha, \beta}\right)^{2}=\operatorname{Id}_{\mathbb{C}^{2}}$ if and only if $\beta=\rho$ and $\alpha \in \mathbb{R} \beta$.

\subsection{Notation}

The Euclidean norm on $\mathbb{C}^{2}=\mathbb{R}^{4}$ will be denoted \|\| . We denote by $H$ the map $H_{\alpha, \beta}$, where $\beta=e^{i \theta}$ for some $\theta \in \mathbb{R}$ with $-\pi<\theta \leq \pi$, and $\alpha=r \beta$ for some $r \in \mathbb{R}$. The involutions $R_{\beta}$ and $R_{\beta} H$ will be denoted by $R$ and $U$, respectively. Thus $H$ is $R$-reversible, $H=R U$ and

$$
U((z, w))=\left(\beta \bar{z}, \beta \bar{\alpha}-\bar{w}-\beta \bar{z}^{2}\right) .
$$

Here, $\beta \bar{z}$ is obtained from $z$ by reflection in the line inclined at $\frac{\theta}{2}$ to the real axis. We call this line the $U$-line. For $n \in \mathbb{Z}, H^{-n}((0,0))=U H^{n-1}((0,0))$, so the projection onto the $z$-plane of $\operatorname{orb}((0,0))$ is symmetrical about the $U$-line. This symmetry can be observed in Figure 1 .

The space of parameters for which $H$ is $R$-reversible is $\mathcal{P}:=\{(\alpha, \beta):|\beta|=1, \alpha \in \mathbb{R} \beta\}$. If $r>0$, $-\pi<\theta \leq \pi$ and $\alpha=r e^{i \theta}$, then $\alpha$ determines two points $\operatorname{pos}(\alpha):=\left(r e^{i \theta}, e^{i \theta}\right)$ and $\operatorname{neg}(\alpha):=\left(r e^{i \theta},-e^{i \theta}\right)=$ $\left(-r e^{i(\theta \pm \pi)}, e^{i(\theta \pm \pi)}\right)$ in $\mathcal{P}$.

For $P \in \mathbb{C}^{2}$, we say that $P$ is periodic of order $n$ if $n$ is the least positive integer such that $H^{n}(P)=P$. The set of periodic points of a given order $n$ is invariant under both $R$ and $U$. A periodic point $P$ is $U$-symmetric, resp. $R$-symmetric, if $U(P)=P$, respectively $R(P)=P$.

\section{FIXED POINTS}

\subsection{Symmetry}

Let

$$
f(z)=z^{2}+(\beta+1) z-\alpha .
$$

For $P=(z, w) \in \mathbb{C}^{2}$,

$$
H(P)=P \Leftrightarrow w=z \text { and } f(z)=0 .
$$

Counting multiplicity, there are two fixed points determined by the zeros of $f$. Let $P$ be a fixed point for $H$. Then $R(P)=U(P)$ is a fixed point. If $P=R(P)=$ $U(P)$, we shall say that $P$ is symmetric.

Theorem 3.1. The fixed points of $H$ are symmetric if and only if $r \geq-c^{2}$, where $c=\cos \frac{\theta}{2}$.

Proof: The fixed points have the form $(z, z)$, where $f(z)=0$, and, as $U((z, z))$ is also fixed, $U((z, z))=$ 
$(\beta \bar{z}, \beta \bar{z})$. For $v \in \mathbb{C}$,

$$
f\left(v e^{\frac{i \theta}{2}}\right)=\beta\left(v^{2}+2 c v-r\right) .
$$

Thus the zeros of $f$ are $z=v e^{\frac{i \theta}{2}}$, where $v=-c \pm \sqrt{c^{2}+r}$. The fixed points are symmetric if and only if these are on the $U$-line if and only if $r \geq-c^{2}$.

\subsection{Ellipticity}

We now analyse the fixed points identified in Section 3.1 in terms of local dynamics. Let $P=(z, w) \in \mathbb{C}^{2}$, let $n$ be a positive integer, and let $J_{n}(P)$ denote the Jacobian matrix of $H^{n}$ at $P$. Then

$$
J_{1}(P)=\left(\begin{array}{cc}
-2 z & -\beta \\
1 & 0
\end{array}\right) \text { and } \operatorname{det} J_{1}(P)=\beta .
$$

Let $P$ be periodic of order $n$. By $(3-4),\left|\operatorname{det} J_{1}(P)\right|=1$, so $\left|\operatorname{det} J_{n}(P)\right|=1$. Either both eigenvalues of $J_{n}(P)$ have modulus 1 , in which case $P$ is elliptic, or one has modulus $>1$ and the other has modulus $<1$, in which case $P$ is a saddle point. The dynamics at saddle points is well understood in terms of the stable and unstable manifolds, e.g., [Bedford et al. 91, Fornæss 96, Smillie and Buzzard 97]. If $n \in \mathbb{N}$, then

$$
R H^{n} R=H^{-n}=U H^{n} U \text { and } U H^{-n}=H^{n-1} R .
$$

Hence the stable and unstable manifolds are mapped to each other by $R$ and by $U$. In a sense made precise in [Bedford et al. 93] or [Smillie and Buzzard 97, Corollary 13.4], most periodic points are saddle points.

Theorem 3.2. Let $c=\cos \frac{\theta}{2}$.

(i) If $r<-c^{2}$, that is, if the fixed points of $H$ are not symmetric, then they are saddle points.

(ii) If $-c^{2} \leq r \leq 1-2 c$, then both fixed points are elliptic.

(iii) If $1-2 c<r \leq 1+2 c$, then one fixed point is elliptic and one is a saddle point.

(iv) If $r>1+2 c$, then both fixed points are saddle points.

Proof: Using (3-4), one shows that, for all $w, z \in \mathbb{C}$, the eigenvalues of $J_{1}((z, w))$ are $\lambda_{1}(z)=-z+\sqrt{z^{2}-\beta}$ and $\lambda_{2}(z)=-z-\sqrt{z^{2}-\beta}$. Let $z=v e^{\frac{i \theta}{2}}, v \in \mathbb{C}$. Then $\lambda_{1}(z), \lambda_{2}(z)=e^{\frac{i \theta}{2}}\left(-v \pm \sqrt{v^{2}-1}\right)$. Hence $\left|\lambda_{1}(z)\right|=1=$ $\left|\lambda_{2}(z)\right|$ if and only if $v \in \mathbb{R}$ and $v^{2} \leq 1$.

By Theorem 3.1 and its proof, the fixed points have the form $(z, z)$, where $z=\left(-c \pm \sqrt{c^{2}+r}\right) e^{\frac{i \theta}{2}}$. (i)-(iv) follow easily.

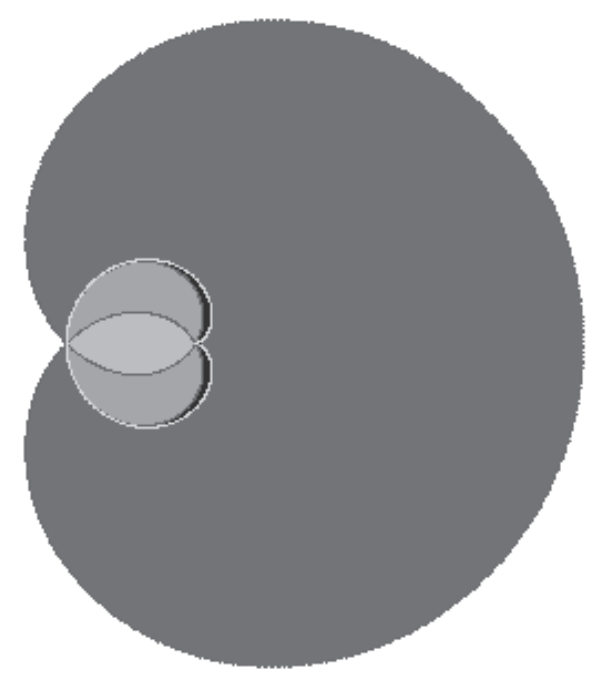

FIGURE 2. The regions where fixed points are elliptic.

Remark 3.3. Corresponding to $0 \neq \alpha \in \mathbb{C}$, there are four fixed points, $P_{1}^{+}$and $P_{2}^{+}$for $\operatorname{pos}(\alpha)$, and $P_{1}^{-}$and $P_{2}^{-}$for neg $(\alpha)$. Number these so that $\left\|P_{1}^{+}\right\| \geq\left\|P_{2}^{+}\right\|$ and $\left\|P_{1}^{-}\right\| \geq\left\|P_{2}^{-}\right\|$. Figure 2 shows the values of $\alpha$, determined by Theorem 3.2, for which there are elliptic fixed points. These are $P_{2}^{+}$everywhere that is shaded, $P_{2}^{-}$everywhere except in the large outermost region, $P_{1}^{+}$ in the eye where the shading is lightest and $P_{1}^{-}$in the darkest regions.

\subsection{Linearization}

We now discuss orbits for the linearization $L_{H}$ of $H$ in the case where the fixed points are elliptic. This will provide a basis for discussions of orbits of $H$ later in the paper. Thus

$$
L_{H}((z, w))=(-2 \zeta z-\beta w, z)=\left(M(z, w)^{T}\right)^{T},
$$

where $P=(\zeta, \zeta)$ is an elliptic fixed point and $M=J_{1}(P)$. By Theorem 3.2 and its proof, the eigenvalues of $M$ can be written in the form $\lambda_{1}=e^{i\left(\frac{\theta}{2}+\phi\right)}$ and $\lambda_{2}=e^{i\left(\frac{\theta}{2}-\phi\right)}$ for some $\phi \in \mathbb{R}$. If both $e^{i \theta}$ and $e^{i \phi}$ are roots of unity, then $L_{H}$ has finite order and hence $H$ cannot be locally conjugate to $L_{H}$. A condition on the eigenvalues under which $L_{H}$ is locally conjugate to $H$ is given by [Zehnder 77].

Suppose that $\beta$ is a primitive $m$ th root of unity, but that $e^{i \phi}$ is not a root of unity. Then $\lambda_{2}^{m}=e^{-i m\left(\frac{\theta}{2}+\phi\right)}=$ $\lambda_{1}^{-m}$. Let

$$
D=\left[\begin{array}{cc}
\lambda_{1} & 0 \\
0 & \lambda_{2}
\end{array}\right]
$$




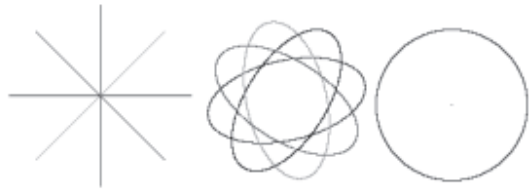

FIGURE 3. Projections of orbits for $L_{H}$ with $\theta=\pi / 4$.

If $z_{1} z_{2} \neq 0$, the orbit of $\left(z_{1}, z_{2}\right)$ for the action of the group $\left\langle D^{m}\right\rangle$ lies on, and is dense in, the closed curve $\left\{\left(e^{i t} z_{1}, e^{-i t} z_{2}\right)\right\}$ and its orbit for the action of $\langle D\rangle$ is dense in the union of $m$ such closed curves. Projections of such curves onto a complex line $\rho z_{1}+\sigma z_{2}=0$ are (possibly degenerate) ellipses. The orbit of $\left(z_{1}, 0\right)$ or $\left(0, z_{2}\right)$ for the action of $\langle D\rangle$ is dense in a circle $\left\{\left(e^{i t} z_{1}, 0\right)\right\}$ or $\left\{\left(0, e^{-i t} z_{2}\right)\right\}$, in the $z_{1}$ - or $z_{2}$-plane. Consequently, for $(z, w) \in \mathbb{C}^{2}$, the orbit for the action of $\left\langle L_{H}\right\rangle$ is dense either in the union of $m$ (possibly degenerate) closed curves, whose projections onto the $z$-plane are ellipses, or in a single such curve. Examples of some orbits for $L_{H}$ are shown in Figure 3.

If the eigenvectors generate a free abelian subgroup of $\mathbb{C}^{*}$ of rank 2 , then orbits for the action of $\langle D\rangle$ are dense in two-dimensional tori $\left\{\left(e^{i t} z_{1}, e^{i u} z_{2}\right)\right\}$, so orbits for the action of $\left\langle L_{H}\right\rangle$ are also dense in two-dimensional tori.

\section{PERIODIC POINTS OF ORDER 2}

\subsection{Symmetry}

For $P=(z, w) \in \mathbb{C}^{2}$, if $\beta \neq-1$,

$$
H^{2}(P)=P \Leftrightarrow(1+\beta) w=\alpha-z^{2} \text { and } f(z) g(z)=0,
$$

where $f(z)$ is as in $(3-1)$ and

$$
g(z):=z^{2}-(\beta+1) z+(1+\beta)^{2}-\alpha .
$$

Counting multiplicity, this determines the four points $P$ such that $H^{2}(P)=P$, including the fixed points. Thus there are at most two periodic points of order 2 . If $P$ is a periodic point of order 2, then so are $H(P), R(P)$, and $U(P)$. As $z$ determines $w, P$ is $U$-symmetric if and only if $z$ is on the $U$-line. Also $R(P) \neq U(P)$, otherwise $H(P)=P$, so either $P$ is $R$-symmetric and $U(P)=$ $H(P)$, in which case $R H(P)=U(P)=H(P)$ and $H(P)$ is $R$-symmetric, or $P$ is $U$-symmetric and $R(P)=H(P)$, which is $U$-symmetric.

Theorem 4.1. Let $c=\cos \frac{\theta}{2}$. (i) If $r=3 c^{2} \neq 0$, then $H$ has no periodic points of order 2.

(ii) If $\beta \neq-1$ and $r \neq 3 c^{2}$, then $H$ has precisely two distinct periodic points of order 2. These are $U$ symmetric if $r>3 c^{2}$ and are $R$-symmetric if $r<$ $3 c^{2}$.

(iii) If $\beta=-1$ and $r \neq 0$, then the periodic points of order 2 are the two points of the form $(z,-z)$ where $z^{2}=\alpha$.

Proof: For $v \in \mathbb{C}, g\left(v e^{\frac{i \theta}{2}}\right)=0 \Leftrightarrow v^{2}-2 c v+4 c^{2}-r=0$ so the zeros of $g$ are $z=v e^{\frac{i \theta}{2}}$ where $v=c \pm \sqrt{r-3 c^{2}}$.

(i) Suppose that $r=3 c^{2} \neq 0$. Then $\beta \neq-1$ and the double zero $c e^{\frac{i \theta}{2}}$ of $g$ is, by $(3-3)$, a zero of $f$. For periodic points of order 1 or $2, w$ is determined by $z$ so the solutions of $H^{2}((z, w))=(z, w)$ are already solutions of $H((z, w))=(z, w)$.

(ii) If $\beta \neq-1$ and $r \neq 3 c^{2}$, then $g$ and $f$ have no common zero so $H$ has two periodic points, $\left(z_{1}, w_{1}\right)$ and $\left(z_{2}, w_{2}\right)$, say, of order 2 , with $z_{1}, z_{2}=v e^{\frac{i \theta}{2}}$, where $v=$ $c \pm \sqrt{r-3 c^{2}}$. The result follows.

(iii) This is routine.

\subsection{Ellipticity}

Theorem 4.2. With $c$ as in Theorem 4.1, if $r \neq 3 c^{2}$, then the periodic points of $H$ of order 2 are elliptic if and only if $4 c^{2}-1 \leq r \leq 4 c^{2}$.

Proof: Let $\left\{\left(z_{i}, w_{i}\right): i=1,2\right\}$ be an orbit of period 2 under $H$. The Jacobian matrix of $H^{2}$ at $\left(z_{i}, w_{i}\right)$ has trace $t=4 z_{1} z_{2}-2 \beta$ and determinant $d=\beta^{2}$. As $z_{1}$ and $z_{2}$ are the roots of $g, t=4(1+\beta)^{2}-4 \alpha-2 \beta=2 \beta b$, where $b=8 c^{2}-2 r-1$. Hence $t^{2}-4 d=t^{2}-4 \beta^{2}=$ $4 \beta^{2}\left(b^{2}-1\right)$. The eigenvalues are $\beta\left(-b \pm i \sqrt{1-b^{2}}\right)$ and these have modulus 1 if and only if $b^{2}-1 \geq 0$, that is if and only if $4 c^{2}-1 \leq r \leq 4 c^{2}$.

\subsection{Notation}

For $n \geq 1$, let $Q_{n}$ denote the set of all $(\alpha, \beta) \in \mathcal{P}$ for which $H$ has an elliptic periodic point of order $n$. In the notation of Section 2.1, Figure 4 shows, in darker shading, respectively lighter shading, the values of $\alpha$ for which $\operatorname{pos}(\alpha) \in Q_{2}$, respectively $\operatorname{neg}(\alpha) \in Q_{2}$.

\subsection{Points of Period 3}

If $P$ is periodic of order 3, then $H U(P)=R(P)$ so $R(P)$ and $U(P)$ must be in the same orbit. If there is 


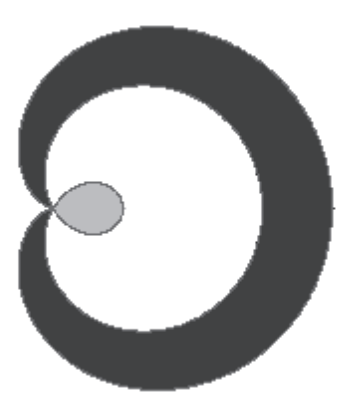

FIGURE 4. The set $Q_{2}$.

a symmetric periodic point of order 3 , for either $R$ or $U$, then there is an orbit of the form $\left\{P, H(P), H^{2}(P)\right\}$ where $P$ is $R$-symmetric, $H(P)$ is $U$-symmetric, and $U(P)=H^{2}(P)=R(H(P))$. Call such an orbit symmetric. If there is no symmetric orbit then, for any periodic point $P$ of order $3, \operatorname{orb}(U(P))=\operatorname{orb}(R(P)) \neq \operatorname{orb}(P)$.

For calculation of the periodic points $(z, w)$ of order 3 , there is a polynomial $h$, of degree 6 , such that, with $f$ as in $(3-1)$, the zeros of $h f$ determine the $z$ coordinates of the eight points, up to multiplicity, where $H^{3}(z, w)=(z, w)$. Except when $2 \beta z^{2}+\beta^{4}-2 \alpha \beta+1=0$, $z$ determines $w$.

Following a suggestion of the referee, we have used the method described in [Giarrusso and Fisher 95] to factorize $h$ as the product of the two cubics

$$
\begin{array}{r}
z^{3}-\Omega z^{2}-\left(\alpha+\beta^{2}-(\beta+1) \Omega-\beta+1\right) z \\
-\alpha(\beta+1-\Omega)+\beta^{3}-\beta \Omega+1
\end{array}
$$

where $\Omega$ represents the sum of the $z$-coordinates of the three points, $\left(z_{i}, w_{i}\right), 1 \leq i \leq 3$, in an orbit of period 3 and is a root of the quadratic

$$
\Omega^{2}-(\beta+1) \Omega+2 \beta^{2}+2-2 \beta-\alpha .
$$

At each of these points, the Jacobian matrix $J_{3}$ has trace $-8 z_{1} z_{2} z_{3}+2 \beta\left(z_{1}+z_{2}+z_{3}\right)=-8\left(\alpha(\beta+1-\Omega)-\beta^{3}+\right.$ $\beta \Omega-1)+2 \beta \Omega$, and determinant $\beta^{3}$. Writing $z=v e^{\frac{i \theta}{2}}$, $\Omega=\Gamma e^{\frac{i \theta}{2}}$ and $c=\cos \frac{\theta}{2}$, the roots of $(4-3)$ have the form $v e^{\frac{i \theta}{2}}$ where

$v^{3}-\Gamma v^{2}-\left(r+4 c^{2}-3-2 \Gamma c\right) v+\Gamma r-\Gamma-2 r c+8 c^{3}-6 c=0$

and

$$
\Gamma=c \pm \sqrt{6+r-7 c^{2}} .
$$

The eigenvalues of $J_{3}\left(z_{i}, w_{i}\right)$ are $e^{\frac{3 i \theta}{2}}\left(u \pm \sqrt{u^{2}-1}\right)$, where $u=(4 r-3) \Gamma-8 c(r+3)+32 c^{3}$.

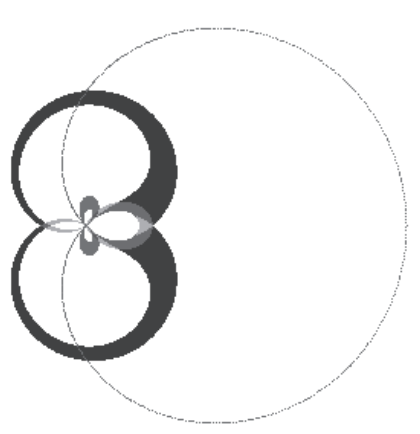

FIGURE 5. The set $Q_{3}$.

If $6+r \geq 7 c^{2}$, each of the cubics in (4-5) has a real root so each orbit of period 3 contains a point $(z, w)$ with $z$ on the $U$-line. In the situation where $z$ determines $w$, such a point must be $U$-symmetric. In the exceptional case, a lengthy calculation shows that the only examples of periodic points $(z, w)$ of order 3 that are not $U$-symmetric, but for which $z$ is on the $U$-line, occur with $\beta=1$ and $\alpha \geq 1$, in which case $\{(z, z),(1-z, z),(z, 1-z)\}$ is a symmetric orbit, with $U((1-z, z))=(1-z, z)$, when $z^{2}=\alpha-1$. Therefore, if $6+r \geq 7 c^{2}$, there are two symmetric orbits of order 3 .

The points in the orbit of period 3 determined by either value of $\Gamma$ are elliptic if $u \in \mathbb{R}$ and $u^{2} \leq 1$. Note that $u \in \mathbb{R}$ if either $6+r \geq 7 c^{2}$ or $r=\frac{3}{4}$. In the latter case, there is a nonsymmetric orbit of elliptic points of order 3 when $28 c^{2}>27$ and $-1 \leq 32 c^{3}-30 c \leq 1$. When $6+r=7 c^{2}$, there is a symmetric orbit $\mathcal{O}$ of period 3 and multiplicity 2 . Here $u=\cos \frac{3 \theta}{2}$ so the points in $\mathcal{O}$ are elliptic.

Points $\alpha$ for which there are elliptic points of period three, for $\operatorname{pos}(\alpha)$ or neg $(\alpha)$, are shown, with various combinations indicated by different levels of shading, in Figure 5 .

\subsection{Elliptic Periodic Points and the Keep Set}

The forward and backward keep sets $K^{+}$and $K^{-}$of $H$ are defined as follows:

$$
\begin{aligned}
& K^{+}=\left\{P \in \mathbb{C}^{2}:\left\{H^{n}(P)\right\}_{n>0} \text { is bounded }\right\} \\
& K^{-}=\left\{P \in \mathbb{C}^{2}:\left\{H^{n}(P)\right\}_{n<0} \text { is bounded }\right\}
\end{aligned}
$$

For example, see [Hubbard and Oberste-Vorth 94]. The keep set $K$ is $K^{+} \cap K^{-}$. By $(3-5)$, for $P \in \mathbb{C}^{2}$,

$$
P \in K^{+} \Leftrightarrow R(P) \in K^{-} \Leftrightarrow U(P) \in K
$$

Hence $K$ is invariant under both $U$ and $R$ and if $P$ is fixed by either $R$ or $U$, then $P \in K^{+} \Leftrightarrow P \in K$. 
It is known, e.g., [Smillie and Buzzard 97, Theorem 13.2] that periodic saddle points must be on the boundary of $K$, so any periodic point $P \in \operatorname{Int} K$ must be elliptic. We thank the referee for pointing out that, at any such point, $H$ is locally conjugate to its linearization. On a dense subset of $\mathcal{P}$, containing those points $(\alpha, \beta)$ where the eigenvalues of the Jacobian matrix $J_{1}$ are roots of unity at the fixed points, $H$ cannot be locally conjugate to $L_{H}$ at the fixed points, which are therefore not in Int $K$.

Experiments investigating whether selected points close to periodic points of orders 1,2 , or 3 are in the keep set produce pictures remarkably similar to those in Figures 2, 4 and 5. It would be interesting to know for which elliptic periodic points $P$ there exists a neighbourhood $U$ of $P$ such that $U \backslash K$ has measure zero. We would also be interested to know more about the sets $Q_{n}$ and their union $Q$. In particular, how does the Lebesgue measure of $Q_{n}$ behave as $n$ increases and is the Lebesgue measure of $Q$ finite? Or could $Q$ be the whole of $\mathcal{P}$ ?

\section{DYNAMICAL RELATIONS}

The dynamics of the Hénon map are known to be similar to those of the horseshoe map, see [Smillie and Buzzard 97, Section 5] or [Oberste-Vorth 97, Section 4]. For the reversible Hénon maps considered here, it is possible to be precise about the bounds which occur.

Definition 5.1. For $0 \neq \alpha \in \mathbb{C}$, let

and let

$$
b_{\alpha}=1+\sqrt{1+|\alpha|} \in \mathbb{R}
$$

$$
\begin{aligned}
V & =\left\{(z, w):|z| \leq b_{\alpha} \text { and }|w| \leq b_{\alpha}\right\} \\
V^{+} & =\left\{(z, w):|w|>b_{\alpha} \text { and }|w| \geq|z|\right\} \\
V^{-} & =\left\{(z, w):|z|>b_{\alpha} \text { and }|z| \geq|w|\right\} .
\end{aligned}
$$

We note that, for the involution $R$ defined in (1-2), $R\left(V^{+}\right)=V^{-}, R\left(V^{-}\right)=R\left(V^{+}\right)$and $R(V)=V$.

\section{Proposition 5.2.}

(i) $H\left(V^{-}\right) \subseteq V^{-}$.

(ii) If $P \in V^{-}$then $\left\|H^{n}(P)\right\| \rightarrow \infty$ as $n \rightarrow \infty$.

(iii) $H^{-1}\left(V^{+}\right) \subseteq V^{+}$.

(iv) If $P \in V^{+}$then $\left\|H^{-n}(P)\right\| \rightarrow \infty$ as $n \rightarrow \infty$.

(v) $H(V) \subseteq V \cup V^{-}$and $H^{-1}(V) \subseteq V \cup V^{+}$.

(vi) $K \subseteq V$.
Proof: Note that

$$
|\alpha| b_{\alpha}^{2}-b_{\alpha}-1=b_{\alpha} .
$$

(i) Let $(z, w) \in V^{-}$and let $(u, v)=H((z, w))$. Then $|z| \geq|w|$ and $|z| \geq b_{\alpha}(1+\epsilon)$ for some $\epsilon>0$. Now

$$
\frac{|u|}{|z|} \geq \frac{1}{|z|}\left(|z|^{2}-|w|-|\alpha|\right) \geq|z|-1-\frac{|\alpha|}{|z|} .
$$

Using (5-5),

$$
\begin{aligned}
|z|-1-\frac{|\alpha|}{|z|} & \geq b_{\alpha}(1+\epsilon)-1-\frac{|\alpha|}{b_{\alpha}(1+\epsilon)} \\
& =\frac{\left(\epsilon^{2}+2 \epsilon\right) b_{\alpha}-\epsilon+1}{(1+\epsilon)} \geq 1+2 \epsilon .
\end{aligned}
$$

Thus $|u|>|z|>b_{\alpha}$ and $|v|=|z| \leq|u|$ and so $(u, v) \in V^{-}$.

(ii) Let $P=(z, w)$ and $\left(u_{n}, v_{n}\right)=H^{n}((z, w))$. If $(z, w) \in V^{-}$, the above argument shows that $\left|v_{n+1}\right|=$ $\left|u_{n}\right| \geq(1+2 \epsilon)^{n}|z|$ for some $\epsilon>0$.

(iii) Using (3-5), $H^{-1}\left(V^{+}\right)=R H R\left(V^{+}\right)=$ $R H\left(V^{-}\right) \subseteq R\left(V^{-}\right)=V^{+}$.

(iv) follows from (ii) and (3-5), while (v) and (vi) are immediate from (i)-(iv).

Remark 5.3. The bound $b_{\alpha}$ is, in a sense, best possible, for if $\alpha=\beta=-1$, then $(z, w)=(-1-\sqrt{2}, 1+\sqrt{2})$ is a fixed point of $H$ and $b_{\alpha}=1+\sqrt{2}$.

\section{ORBITS}

Most of this section is concerned with experimental observations of orbits of $(0,0)$. Orbits in Int $K$, for a class of volume-preserving maps including $H$, are discussed in [Bedford et al. 91, Appendix] where it is shown that the closure of the orbit of a generic point is a union of $q k$ dimensional tori for $k=1$ or $k=2$. From Section 3.3 and our experimentation, it appears that if $\beta$ is a primitive $m$ th root of unity and $r$ is small, then $k=1$ and $q=m$. However, it appears that, for larger $r, q$ can be $n m$ for an integer $n>1$. In Section 6.3, we shall describe an example where $m=25$, but $q$ appears to be 1075 . If $\beta$ is not a root of unity, then $k$ may be 2 and, although $q=1$ for the linearization and for small $r$, experimentation suggests that $q$ need not always be 1. Examples with $q>1$ will be observed in Section 6.2 and Section 6.3 .

We restrict our study to the case where $\alpha=r e^{i \theta}$ with $r>0$ so that $\alpha$ determines $H$. The observations below are based on a Java applet, available 


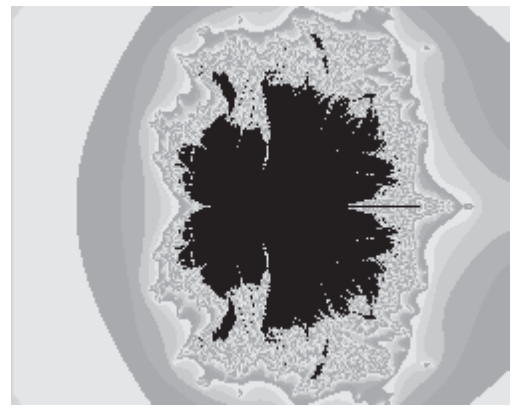

FIGURE 6. The set $B$.

at http://www.shef.ac.uk/ ${ }^{\sim}$ daj/henon/H.html, which, given $r$ and $\theta$, plots the $z$-projection $\Pi_{z}(\operatorname{orb}((0,0))$. The set $B:=\{\alpha:(0,0) \in K\}$ is shown in Figure 6 . This was plotted using a Java applet based on the bound $b_{\alpha}$ from Proposition 5.2(ii). Note that if $|\alpha|>3$, then $b_{\alpha}<|\alpha|$ and hence, since $H((0,0))=(\alpha, 0) \in V^{-},(0,0) \notin K$.

\subsection{Coset Orbits}

Let $i$ and $k$ be integers with $0 \leq i<k$. The subset $\left\{H^{j k+i}((0,0)): j \in \mathbb{Z}\right\}$ of $\operatorname{orb}((0,0))$ will be denoted $\mathcal{O}_{i}^{j}$ and will be called the $i$-th coset orbit for the subgroup $\left\langle H^{k}\right\rangle$.

Suppose that $\beta$ is a primitive $m$ th root of unity. Recall from Section 3.3 that the orbit of a generic point under $\left\langle L_{H}\right\rangle$ is dense in a union of $m$ closed curves whose $z$-projections are ellipses. For small $r, \Pi_{z}(\operatorname{orb}((0,0)))$ appears to be dense in the union of $m$ ovals, each corresponding to one of the coset orbits $\mathcal{O}_{i}^{m}$. In Figure 7, where $\theta=\frac{\pi}{3}, \frac{\pi}{2}, \frac{\pi}{3}$ and $m=6,4,3$, respectively, and in Figure 8 , where $\theta=\frac{\pi}{2}$ and $m=4$, each coset orbit is shaded differently.

As $r$ increases towards the boundary of $B$, the $m$ ovals lose their convexity and smoothness, but remain closed curves. For example, see the top two coset orbits in Figure 14.

Where the line $\left\{x e^{i \theta}: x \geq 0\right\}$ crosses the boundary of $B$, the closed curves are distorted ovals for $\alpha$ close to the boundary, but closer to their original oval shape away from the boundary. Figure 9 shows the coset orbits $\mathcal{O}_{3}^{4}$ for
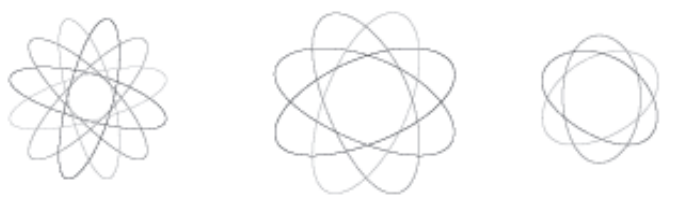

FIGURE 7. Orbits for $m=6,4,3$ respectively.

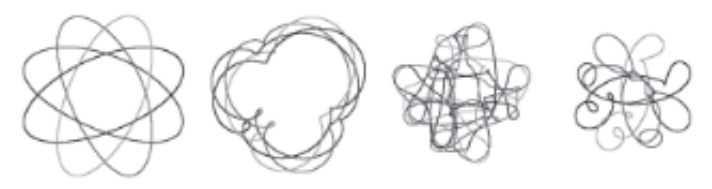

FIGURE 8. Orbits for $r=0.1,0.24,0.246,0.249 ; \theta=\frac{\pi}{2}$.

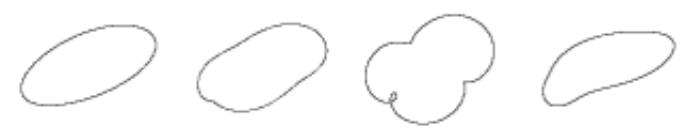

FIGURE 9. Coset orbits for $r=0.1,0.23,0.24,0.3 ; \theta=\frac{\pi}{2}$.

$r=0.1,0.23,0.24,0.3$, and $\theta=\pi / 2$. When $r=0.2462$, the orbit is unbounded. However, the orbit appears to be bounded for $r=0.24853$ (see Figure 10) and has a reasonably simple shape for $r=0.3$.

For fixed small $r$, the eccentricity of the ovals decreases with $\theta$. This can be seen in Figure 7 . If $\beta$ is not a root of unity, the pictures generated by the applet are consistent with orb $((0,0))$ being dense in a finite union of two-dimensional tori. For example, see the orbits in Figure 1.

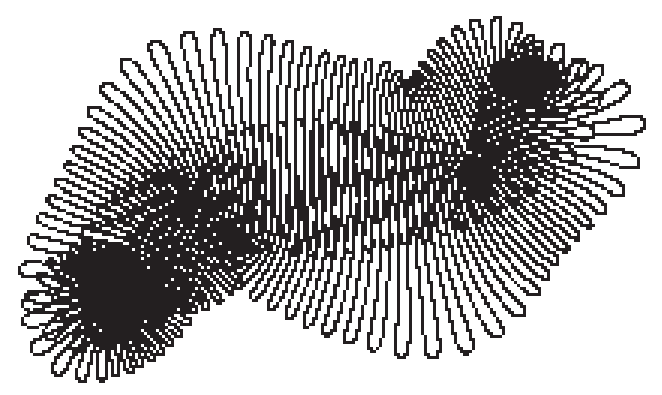

FIGURE 10. A coset orbit for $r=0.24853$ and $\theta=\frac{\pi}{2}$.

\subsection{Islands}

It seems likely that the boundedness of the orbits discussed above is influenced by elliptic fixed points close to the centre of the ovals. Orbits of points close to this fixed point are similar in shape to those of $(0,0)$. There are points on the set $B$ where $\operatorname{orb}((0,0))$ appears to be influenced by elliptic periodic points of order greater than one. For example, for the point $\alpha=e^{\pi i / 3}$, which appears to be on the boundary of an "island" of $B,(0,0)$ is an elliptic periodic point of order 4. For other val- 


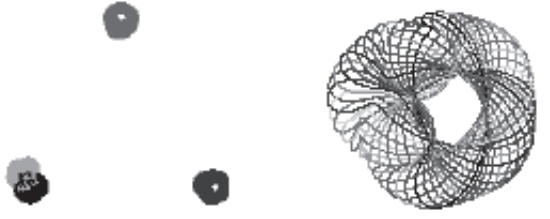

FIGURE 11. Four coset orbits with a close-up of one.

ues of $\alpha$ on this island, the orbits of $(0,0)$ appear to be influenced by such periodic points. Figure 11 shows $\operatorname{orb}((0,0))$ when $r=1$ and $\beta=e^{0.32 \pi i}$, a primitive 25 th root of unity. On the left are the four coset orbits for $H^{4}$ with a close up of one of these, decomposed as the union of 25 coset orbits for $H^{100}$, on the right. This suggests that there may be an orbit $\left\{P_{1}, P_{2}, P_{3}, P_{4}\right\}$ of elliptic periodic points of order 4 , such that there exist neighbourhoods $N\left(P_{1}\right), N\left(P_{2}\right), N\left(P_{3}\right), N\left(P_{4}\right)$ with orb $((0,0)) \subset$ $\bigcup_{1 \leq i \leq 4} N\left(P_{i}\right)$ and $H\left(N\left(P_{i}\right)\right) \subset N\left(P_{i+1 \bmod 4}\right)$.

Values of the parameters at which we have observed similar behaviour are shown on the left of Table 1. Figure 12 shows orbits for the first three rows of the left hand table.

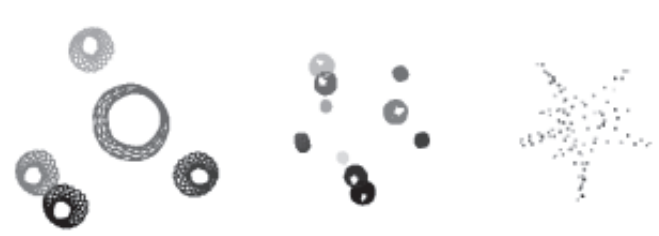

FIGURE 12. Orbits for $\alpha=0.55 e^{\frac{4 \pi i}{9}}, \alpha=0.939 e^{\frac{\pi i}{5}}$, $\alpha=0.9385 e^{0.187 \pi i}$.

\subsection{Bifurcation}

Within the period 4 island, there is some bifurcation. Figure 13 shows the 12 coset orbits for $H^{12}$, where $\alpha=0.98 e^{0.305 \pi i}$, on the left, and $\alpha=0.95 e^{0.3016 \pi i}$, indicating bifurcation from 4 to 12 . The coset orbits for $\alpha=0.98 e^{0.305 \pi i}$ are smoother than those for $\alpha=$ $0.95 e^{0.3016 \pi i}$. Other values of $\alpha$ for which we have observed bifurcation are shown on the right in Table 1.

If $\theta=0.16 \pi$, so that $m=25$, and $r$ is about 0.82 then the coset orbits for $H^{25}$ appear as 25 closed curves. However for $r=0.83$, these each bifurcate into 43 closed curves, suggesting that the closure of $\operatorname{orb}((0,0))$ is the union of 1075 1-dimensional tori. The 11th coset orbits for $r=0.82,0.826$ and 0.83 are shown in Figure 14 .

\begin{tabular}{|l|l|c|}
\hline$r$ & $\theta$ & period \\
\hline 0.55 & $4 \pi / 9$ & 5 \\
\hline 0.939 & $0.2 \pi$ & 11 \\
\hline 0.9385 & $0.187 \pi$ & 116 \\
\hline 0.790666 & $0.295704 \pi$ & 21 \\
\hline 0.788 & $0.36363636 \pi$ & 9 \\
\hline 0.696059 & $0.520135 \pi$ & 10 \\
\hline
\end{tabular}

\begin{tabular}{|l|l|c|}
\hline$r$ & $\theta$ & bifurcation \\
\hline 0.987 & $0.30631 \pi$ & $12 \mapsto 60$ \\
\hline 0.983 & $0.306 \pi$ & $12 \mapsto 444$ \\
\hline 0.943 & $0.19825 \pi$ & $11 \mapsto 99$ \\
\hline 0.962 & $0.198 \pi$ & $11 \mapsto 253$ \\
\hline 0.661 & $0.495 \pi$ & $5 \mapsto 90$ \\
\hline 0.661 & $0.49498971 \pi$ & $90 \mapsto 360$ \\
\hline
\end{tabular}

TABLE 1. Parameters for periodic behaviour and bifurcation.

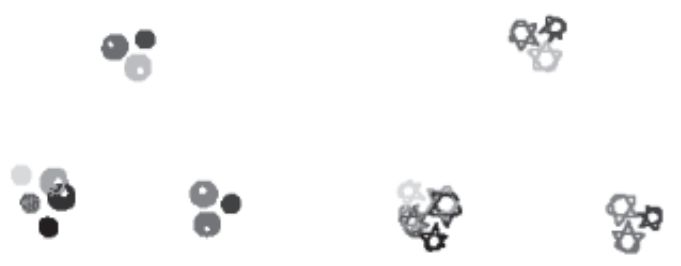

FIGURE 13. Bifurcation into 12.
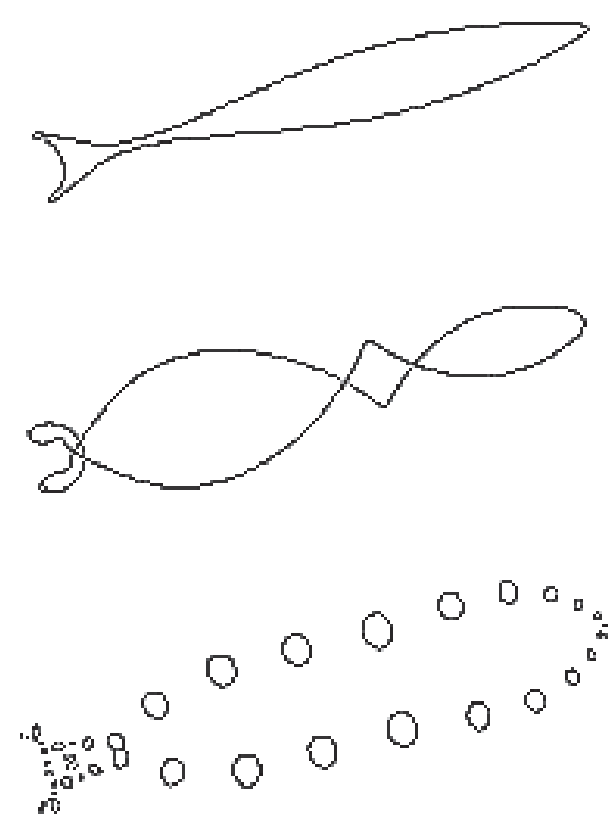

FIGURE 14. Bifurcation of one coset orbit.

\section{REFERENCES}

[Bedford et al. 91] E. Bedford and J. Smillie. "Polynomial diffeomorphisms of $\mathbb{C}^{2}$. II: stable manifolds and recurrence." J. Amer. Math. Soc. 4 (1991), 657-679.

[Bedford et al. 93] E. Bedford, M. Lyubich and J. Smillie. "Distribution of periodic points of polynomial diffeomorphisms of $\mathbb{C}^{2}$." Invent. Math. 114 (1993), 277-288.

[Devaney 76] R. Devaney. "Reversible diffeomorphisms and flows." Trans. Amer. Math. Soc. 218 (1976), 89-113. 
[Devaney 84] R. Devaney. "Homoclinic bifurcations and the area-conserving Hénon map." J. Differential Equations 51 (1984), 254-266.

[Devaney 89] R. Devaney. An Introduction to Chaotic Dynamical Systems, 2nd edition, Addison Wesley, Redwood City, 1989.

[Fornæss 96] J. E. Fornæss. Dynamics in Several Complex Variables. CBMS Regional Conference Series in Mathematics, 87, Amer. Math. Soc., Providence, 1996.

[Friedland et al. 89] S. Friedland and J. Milnor. "Dynamical properties of plane polynomial automorphisms." Ergod. Th.\& Dynam. Sys. 9 (1989), 67-99.

[Giarrusso and Fisher 95] D. Giarrusso and Y. Fisher. "A parameterization of the period 3 hyperbolic components of the Mandelbrot set." Proc. Amer. Math. Soc. 123 (1995), 3731-3737.

[Hale and Koçak 91] J. Hale and H. Koçak. Dynamics and Bifurcations. Springer-Verlag, New York, 1991.
[Jordan 93] D. A. Jordan. "Iterated skew polynomial rings and quantum groups." J. Algebra 156 (1993), 194-218.

[Hubbard and Oberste-Vorth 94] J. H. Hubbard and R. W. Oberste-Vorth. "Hénon mappings in the complex domain I: the global topology of dynamical space." Publ. Math. IHES 79 (1994), 5-46.

[Oberste-Vorth 97] R. W. Oberste-Vorth. "An introduction to multi-dimensional complex dynamics: Hénon mappings in $\mathbb{C}^{2} . "$ Nonlinear analysis, Methods $\&$ Applications 30 (1997), 2143-2154.

[Smillie and Buzzard 97] J. Smillie and G. T. Buzzard. "Complex Dynamics in Several Variables." in Flavors of Geometry, S. Levy, ed., pp. 117-150, Cambridge University Press, 1997.

[Zehnder 77] E. Zehnder. "A simple proof of a theorem by C. L. Siegel." in Geometry and Topology, J.Palis and M.d́lo Carmo, eds., Lecture Notes in Math., vol. 597, pp. 855-866, Springer-Verlag, New York, 1977.

C. R. Jordan, The Open University in Yorkshire, 2 Trevelyan Square, Boar Lane, Leeds LS1 6ED, UK (c.r.jordan@open.ac.uk)

D. A. Jordan, Department of Pure Mathematics, University of Sheffield, Hicks Building, Sheffield S3 7RH, UK (d.a.jordan@sheffield.ac.uk)

J. H. Jordan, Department of Probability and Statistics, University of Sheffield, Hicks Building, Sheffield S3 7RH, UK (jonathan.jordan@sheffield.ac.uk)

Received November 27, 2000; accepted in revised form November 28, 2001. 